\title{
Species specificity, surface exposure, protein expression, immunogenicity, and participation in biofilm formation of Porphyromonas gingivalis HmuY
}

\author{
Teresa Olczak*, Halina Wójtowicz, Justyna Ciuraszkiewicz and Mariusz Olczak
}

\begin{abstract}
Background: Porphyromonas gingivalis is a major etiological agent of chronic periodontitis. The aim of this study was to examine the species specificity, surface exposure, protein expression, immunogenicity, and participation in biofilm formation of the $P$. gingivalis heme-binding protein HmuY.

Results: HmuY is a unique protein of $P$. gingivalis since only low amino-acid sequence homology has been found to proteins encoded in other species. It is exposed on the cell surface and highly abundant in the outer membrane of the cell, in outer-membrane vesicles, and is released into culture medium in a soluble form. The protein is produced constitutively at low levels in bacteria grown under high-iron/heme conditions and at higher levels in bacteria growing under the low-iron/heme conditions typical of dental plaque. HmuY is immunogenic and elicits high IgG antibody titers in rabbits. It is also engaged in homotypic biofilm formation by P. gingivalis. Anti-HmuY antibodies exhibit inhibitory activity against $P$. gingivalis growth and biofilm formation.

Conclusions: Here it is demonstrated that HmuY may play a significant role not only in heme acquisition, but also in biofilm accumulation on abiotic surfaces. The data also suggest that HmuY, as a surface-exposed protein, would be available for recognition by the immune response during chronic periodontitis and the production of anti-HmuY antibodies may inhibit biofilm formation.
\end{abstract}

\section{Background}

Periodontitis is a complex process affecting tooth-supporting tissues [1]. The pathogenesis of periodontal diseases is largely attributed to localized inflammation, which results from interaction between host and microbial factors [2]. The most common etiological agent of chronic periodontitis is Porphyromonas gingivalis, a Gram-negative anaerobic black-pigmented bacterium [3] . On tooth surfaces, $P$. gingivalis is a constituent of the complex multispecies biofilm known as dental plaque, which has properties of other biofilms found in the human body and in the environment. $P$. gingivalis can also colonize the tissues and cells of the gingival epithelium [4]. The bacterium can not only invade, but also

\footnotetext{
* Correspondence: Teresa.Olczak@biotech.uni.wroc.pl

1 Laboratory of Biochemistry, Faculty of Biotechnology, University of Wroclaw, Tamka 2, 50-137 Wroclaw, Poland

Full list of author information is available at the end of the article
}

accumulate inside gingival epithelial cells $[5,6]$. Recent evidence demonstrates that the effect of periodontitis might have systemic consequences since the bacterium can spread systemically and locate to other tissues [7-10].

Bacteria living in a biofilm have a physiology different from that of planktonic cells and they generally live under nutrient limitation, including that of iron and heme. The uptake of heme as iron and protoporphyrin IX is an important mechanism by which $P$. gingivalis and other pathogenic bacteria obtain these compounds for their survival and their ability to establish an infection $[11,12]$. Gram-negative bacteria utilize outer-membrane receptors to acquire heme from host hemoproteins directly or through a hemophore or lipoprotein and then transport the captured heme into the cell. In the case of P. gingivalis, one of the systems of heme acquisition consists of HmuR and HmuY proteins [12]. HmuR is an outer-membrane TonB-dependent receptor involved in heme trans- 
port through the outer membrane [13-16], whereas $\mathrm{HmuY}$ is a heme-binding lipoprotein associated with the outer membrane of the bacterial cell [17-21]. A detailed characterization of the HmuY-heme complex demonstrated that heme, with a midpoint potential of $136 \mathrm{mV}$, is in a low-spin Fe(III) hexa-coordinate environment [20]. In that report we also identified histidines 134 and 166 as potential heme ligands. Recent crystallographic analysis of the HmuY-heme complex confirmed these data and showed that the protein exhibits a unique structure composed of an all- $\beta$ fold [21]. Our studies also showed that HmuY may be functional in the form of dimers/tetramers $[19,21]$. It seems that dimeric HmuY takes up heme and this leads to tetramerization under occlusion of the heme binding sites. Tetrameric HmuY would protect heme from host scavengers and delivered it to HmuR. On the basis of our mutational analysis of HmuY heme ligands [20], an initial step in heme transfer could involve disruption of only one of the two axial histidine ligands, as found for Serratia marcescens hemophore HasA [22]. Once bound by HmuR, heme is translocated across the outer membrane into the periplasm with the assistance of TonB and further heme transport requires the presence of binding proteins to escort it across the periplasm to the cytoplasm. This step might be performed by other $h m u$ operon proteins, so far not characterized $[17,19]$. HmuY, especially in the form associated with the outer membrane, may also store heme and protect the bacterial cell from damage induced by free hemin.

It is likely that HmuY lipoprotein may play a role not only in heme acquisition, but also in the host pathogen response. Therefore the aim of this study was to analyze the surface exposure and expression of HmuY protein in $P$. gingivalis. In addition, in this report we examined the participation of HmuY protein in biofilm formation.

\section{Results and Discussion}

HmuY is a unique $P$. gingivalis protein

Preliminary studies demonstrated that HmuY shows high identity to proteins identified in several $P$. gingivalis strains [17,19]. Here we compared the amino-acid sequences of putative HmuY homologues deposited in databases. Interestingly, we found that $\mathrm{HmuY}$ is similar to proteins encoded in several different species belonging to the Bacteroidetes phylum, which consists of three classes: Bacteroidetes, Flavobacteria, and Sphingobacteria [23]. The Bacteroidetes class consists of anaerobes which are often found in high numbers in the intestinal tracts of animals and which may infect different human tissues, including periodontal tissues (see Additional file 1). Members of the other two classes are mainly aerobic and abundant in many freshwater and marine systems (data not shown). Bacteria encoding putative HmuY homologues have also been identified in several human patho- gens, including those infecting the oral cavity (see Additional file 1) [24-31]. A characteristic feature of all the HmuY homologues identified in this study is biofilm formation. However, although we found several putative HmuY homologues in a broad range of bacteria, the similarity of the amino-acid sequences of HmuY from Porphyromonas and other species was low (5-47\%) (see Additional file 1). Only between $\mathrm{HmuY}$ proteins encoded within Porphyromonas species was the similarity higher (24-100\%) (see Additional file 1). In addition, only P. gingivalis strains possess both histidines engaged in heme coordination [20,21]. Here we also demonstrated that antibodies against purified HmuY raised in rabbits were highly specific and recognized only this antigen in $P$. gingivalis A7436 and W83 whole-cell lysates compared with a $P$. gingivalis $h m u Y$ deletion mutant strain (TO4) (figure 1), E. coli, or Bacteroides fragilis whole-cell lysates (data not shown).

\section{HmuY is exposed on the surface of $P$. gingivalis cells}

The $\mathrm{N}$ terminus of HmuY shares characteristic features of classical lipoproteins, possessing a signal peptide sequence cleaved off by the signal peptidase II $[19,32]$. After removal of the signal peptide, the $\alpha$-amino group of the $\mathrm{N}$-terminal cysteine is acylated, yielding a mature lipoprotein. Although HmuY association with the outer membrane of the $P$. gingivalis cell was previously demonstrated $[17,19,33]$, the orientation of the protein in the outer membrane was not examined. Bacterial lipoproteins may be located at the cell surface or directed into the periplasmic space. We hypothesized previously that

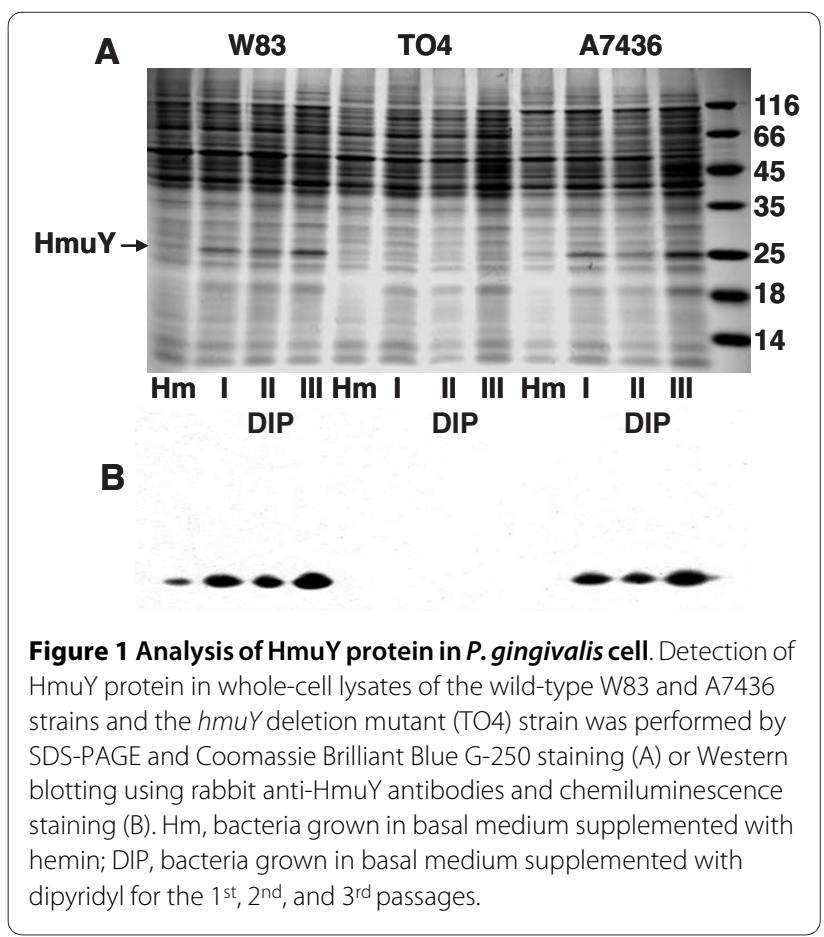


HmuY functions as an external protein [21]. To determine whether HmuY is surface exposed, the proteinase $\mathrm{K}$ accessibility assay was employed using the $P$. gingivalis A7436 and W83 wild-type strains. Upon incubation with proteinase $\mathrm{K}$ of intact cells grown under low-iron/heme conditions, most of the HmuY was not degraded (figure 2A). A similar effect was observed when $P$. gingivalis cells grown under high-iron/heme conditions and E. coli cells over-expressing membrane-associated HmuY were examined (data not shown). It is likely that HmuY may be partially protected by the cell wall, similar to other lipoproteins [34], or resistant to proteinase $\mathrm{K}$ digestion. The latter is highly possible since we previously demonstrated that $\mathrm{HmuY}$ is resistant to the proteolytic action of trypsin and gingipains [21]. Indeed, experiments performed with purified HmuY showed limited degradation of the protein (figure 2A). Therefore we further employed an immunological analysis. Considering the surfaceexposed location of $\mathrm{HmuY}$, the protein attached to the $P$. gingivalis cell should be able to react with antibodies. Dot-blotting analysis showed that rabbit anti-HmuY antibodies, either those present in whole immune serum or a

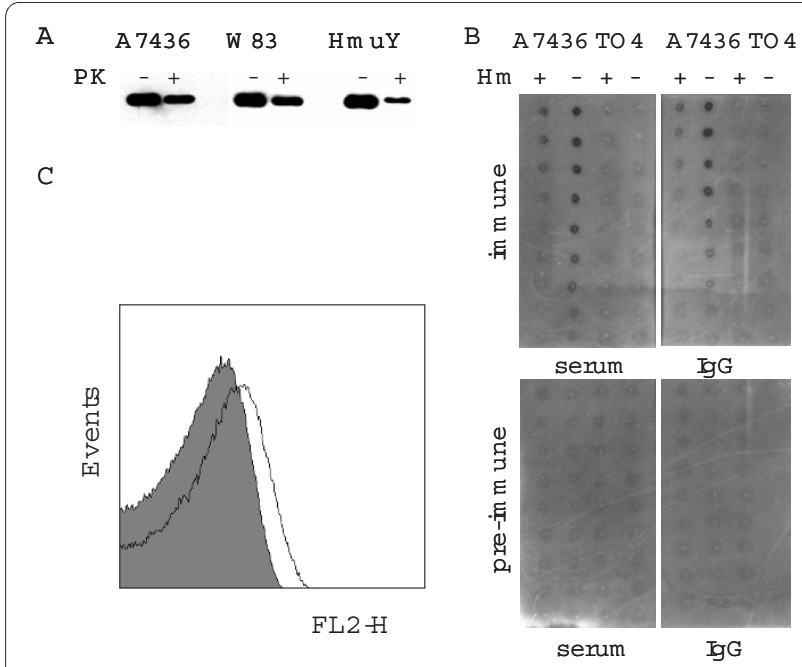

Figure 2 Analysis of surface exposure of $P$. gingivalis HmuY protein. (A) Proteinase K (PK) accessibility assay performed with whole-cell P. gingivalis wild-type A7436 and W83 strains and the hmuY deletion mutant (TO4) grown in basal medium supplemented with dipyridyl and with the purified protein (HmuY). The cells or protein were incubated with proteinase $\mathrm{K}$ at $37^{\circ} \mathrm{C}$ for $30 \mathrm{~min}$ and then analyzed by SDSPAGE and Western blotting. Intact HmuY exposed on the cell surface was analyzed by dot-blotting (B) or FACS (C) analyses. For dot-blotting analysis, varying dilutions of $P$. gingivalis cell suspension (starting at $\mathrm{OD}_{660}=1.0 ; 1 \mu \mathrm{l}$ ) were adsorbed on nitrocellulose membrane and detected with pre-immune serum or purified pre-immune lgGs and immune anti-HmuY serum or purified immune anti-HmuY IgGs. For FACS, P. gingivalis cells were washed and, after blocking nonspecific binding sites, incubated with pre-immune (grey) or anti-HmuY immune serum (transparent). Representative data of the $P$. gingivalis A7436 strain are shown. purified IgG fraction, recognized surface-exposed HmuY with high affinity compared with pre-immune serum or pre-immune IgGs (figure 2B). We did not detect reactivity with anti-HmuY serum or IgGs in the hmuY deletion TO4 mutant cells. A whole-cell ELISA assay highly corroborated that $\mathrm{HmuY}$ is associated with the outer membrane and exposed on the extracellular surface of the cell (see Additional file 2). Since these two experiments were performed using adsorbed cells, FACS analysis was employed to examine free cells in solution. The results shown in figure $2 \mathrm{C}$ confirmed the surface exposure of HmuY protein. Moreover, all these analyses showed that $\mathrm{HmuY}$ is expressed in bacteria grown under low-iron/ heme conditions at higher levels than in bacteria grown under high-iron/heme conditions.

\section{HmuY is one of the dominant proteins produced under low-iron/heme conditions by $\mathrm{P}$. gingivalis}

Previous studies showed that mRNA encoding $\mathrm{HmuY}$ was produced at low levels when bacteria were cultured under high-iron/heme conditions (BM supplemented with hemin), but its production was significantly increased when the bacteria were starved in BM without hemin and supplemented with an iron chelator $[16,17,19]$. To analyze HmuY protein expression in the cell and its release into the culture medium during bacterial growth, Western blotting analysis was employed. We did not detect $P$. gingivalis Fur protein in the culture medium, thus confirming bacterial integrity (data not shown). Similar to mRNA expression, HmuY protein expression was higher in bacteria grown under low-iron/ heme conditions (basal medium without added hemin and supplemented with dipyridyl or human serum) than in bacteria grown under high-iron/heme conditions (figures 1 and 3). During bacterial growth, HmuY was constitutively expressed in the cells of the A7436 strain, reaching similar levels in the cells at the indicated time points (figures 3 and 4). Instead of being degraded by active $P$. gingivalis proteases, constitutively produced $\mathrm{HmuY}$ was accumulated in the culture medium because

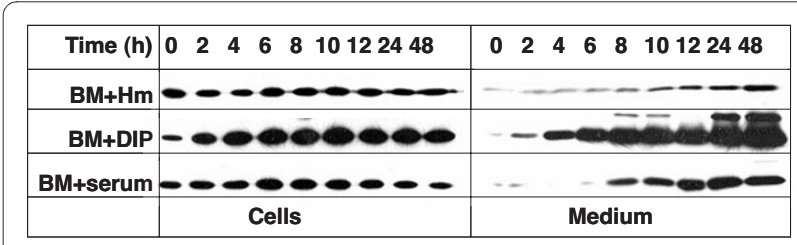

Figure 3 Determination of HmuY expression in $P$. gingivalis grown under various conditions. Bacteria (A7436 strain) were grown in basal medium supplemented with hemin (BM+Hm), $160 \mu \mathrm{M}$ dipyridyl (BM+DIP), or $5 \%$ human serum (BM+serum), collected at the indicated time points, centrifuged, and both cells and culture media analyzed by SDS-PAGE and Western blotting with anti-HmuY antibodies. 


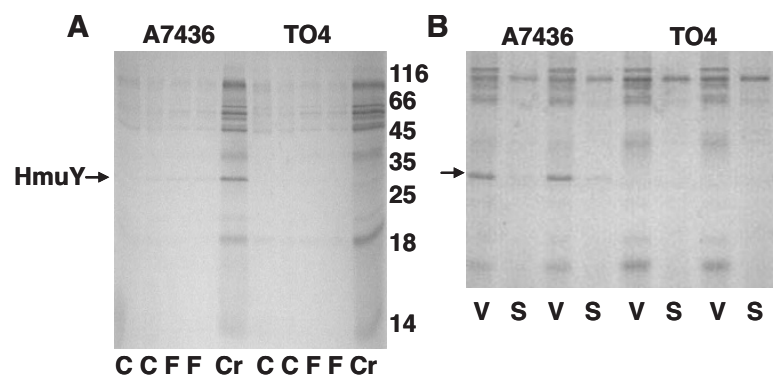

Figure 4 Analysis of HmuY protein in P. gingivalis culture medium. Detection of HmuY protein in whole culture medium (A) or after fractionation of the culture medium by ultracentrifugation (B) of the wild-type A7436 and the hmuY deletion mutant (TO4) strains performed by SDS-PAGE and Coomassie Brilliant Blue G-250 staining. C, culture medium after removal of the cells by centrifugation; $\mathrm{F}$, centrifuged and filtered culture medium; $\mathrm{Cr}$, concentrated culture medium after centrifugation and filtration; $V$, outer-membrane vesicles; $S$, soluble proteins present in culture medium after ultracentrifugation.

during bacterial growth, increasing amounts of the protein were detected in both the outer-membrane vesicleassociated and the soluble form (figures 3 and 4). Our data confirm that the changes observed in gene and protein expression in $P$. gingivalis grown under iron/heme limitation reflect the importance of the environmental levels of these compounds to this bacterium and support the regulation of HmuY expression by iron and heme [19]. The response of P. gingivalis to environmental heme availability was previously mapped on a global scale by transcriptomic analysis using DNA microarrays and by proteomic analysis using mass spectrometry [35-37]. The authors found that mRNA levels of $h m u R$ and $h m u Y$ in the cell significantly increased under heme limitation. In contrast to higher levels of HmuR protein produced under heme limitation in the cell, no significant increase in protein levels of $\mathrm{HmuY}$ was observed under low-heme conditions. The data presented in this study (figures 1, 3, and 4) and earlier [21] demonstrated that HmuY is constitutively expressed and released into the external milieu not only in the form of outer-membrane vesicles, but also in a soluble form, which precluded the protein from being identified as up-regulated in the proteomic analysis.

In contrast, others have shown that $P$. gingivalis enhanced $h m u Y$ mRNA expression in response to low cell density rather than to low iron concentration [38]. The authors found that the expressions of the $h m u Y$ and $h m u R$ genes were highest in $P$. gingivalis grown in the early $\log$ phase, when the cell density is low, but expression levels were significantly decreased in the late log phase, when cell density is much higher. They also suggested that the expression of $h m u Y$ mRNA in P. gingivalis cells grown in the same cell densities was similar regardless of the presence of heme. These results are different from those demonstrating higher $h m u Y$ mRNA expres- sion levels in P. gingivalis cells grown under low-heme conditions and in biofilm, the latter resembling high-celldensity conditions [35-37]. Our results presented in this study corroborate the latter findings and demonstrate that HmuY protein is constitutively produced in the cell at low levels when bacteria are grown under high-iron/ heme conditions; however, significantly higher protein levels are found in cells grown under low-iron/heme conditions, maintained in vitro by the addition of an iron chelator or human serum to the heme-free medium (figure 3). These experiments were performed using $P$. gingivalis cultures grown in the first passage of starvation, thus allowing achieving similar cell densities, especially in the early growth phase (data not shown).

\section{HmuY participates in homotypic biofilm accumulation}

To cope with a changing environment and with continuous attacks of the host antimicrobial defense systems, bacteria produce a biofilm, which plays an important role in chronic infections due to its ability to challenge the host immune system and resist antimicrobial treatment [39]. It has been demonstrated that $P$. gingivalis actively participates in biofilm formation [40], which facilitates the long-term survival of the bacterium and induces an inflammatory reaction that is responsible for the destruction of the hard and soft tooth-supporting tissues. The transition from planktonic bacteria to biofilm-associated cells involves changes in gene expression and is mediated at least in part by intercellular communication. A recent study demonstrated that $\mathrm{HmuY}$ is produced predominantly in P. gingivalis cells grown in biofilm compared with the cells growing in a planktonic form [35]. Biofilm formation begins with the production of an extracellular matrix, a structure that creates a shared space within the cellular community. In prokaryotes, the extracellular matrix is typically composed of carbohydrate polymers and proteins, and many of these proteins possess lipoprotein secretion signals. To determine if HmuY could be engaged in biofilm accumulation, we examined in vitro the homotypic biofilm-forming capabilities of wild-type (A7436, W83, and ATCC 33277) strains and a hmuY deletion mutant constructed in the A7436 strain (TO4). As shown in figure 5 , bacteria grown under low-iron/heme conditions exhibited significantly greater biofilm accumulation than cells grown under high-iron/heme conditions. In addition, our data demonstrated that $\mathrm{HmuY}$ is involved in biofilm formation since $P$. gingivalis cells not producing this protein showed a significantly lower ability to form biofilm (figure 5). In contrast, all P. gingivalis cells grown in a planktonic form exhibited similar growth rates, suggesting that the mutation did not influence bacterial growth (see Additional file 3). All these data suggest that HmuY may play a significant role in biofilm accumulation on abiotic surfaces and support the importance of 


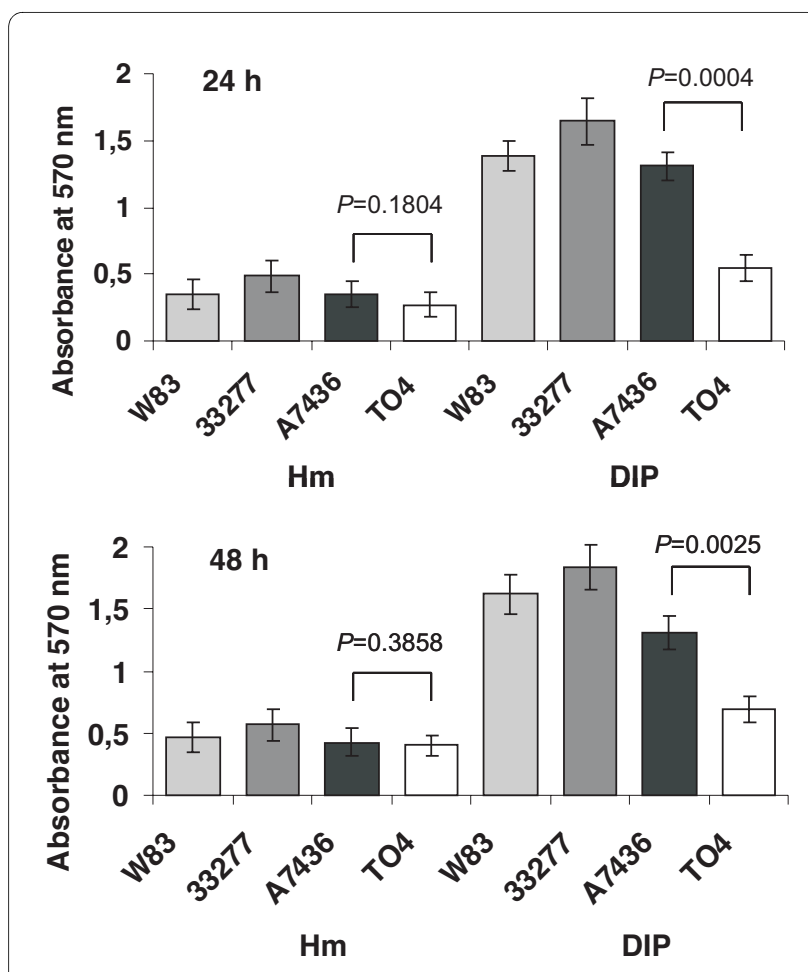

Figure 5 Homotypic biofilm formation by $P$. gingivalis. $P$. gingivalis wild-type (A7436, W83, and ATCC 33277) strains and the hmuY deletion mutant strain constructed in A7436 (TO4) were grown in basal medium supplemented with hemin ( $\mathrm{Hm}$ ) or dipyridyl (DIP). The microtiter plate biofilms were stained with crystal violet. Data are shown as the mean $\pm S D$ of three independent experiments $(n=24)$. Differences between the TO4 mutant and the wild-type A7436 strain expressed as $p$ values are given above the respective bars.

HmuY for P. gingivalis survival during starvation, conditions similar to those found in plaque.

To facilitate adaptation to life within the oral cavity, $P$. gingivalis must be capable of sensing and responding to the prevailing environmental conditions, including nutrient availability, cell density, and the presence of other bacteria. It has been recently shown that $P$. gingivalis possesses the $l u x S$ gene and produces a functional AI-2 autoinducer [41]. In P. gingivalis, among the many different bacterial features that are regulated by quorum sensing using LuxS protein is the expression of genes involved in iron and heme acquisition, including the heme receptor HmuR [41,42]. Although the authors analyzed hmuR gene expression only, it is highly possible that the expressions of other components of $h m u$ operon, such as $h m u Y$, may also be regulated by LuxS signaling. It has been shown that LuxS is also required in P. gingivalis for the development of biofilm under low-heme conditions [43], which additionally supports an involvement of $\mathrm{HmuY}$ in both heme uptake and biofilm accumulation.

\section{Anti-HmuY antibodies inhibit P. gingivalis growth and biofilm accumulation}

We further tested whether anti-HmuY antibodies had inhibitory activity against $P$. gingivalis, which was first determined by measuring the OD at $660 \mathrm{~nm}$ for planktonic bacteria after incubation of bacterial suspensions with pre-immune or immune anti-HmuY IgGs (figure 6). As shown in figure 7, incubation of P. gingivalis wild-type strains with immune anti-HmuY IgGs slightly decreased subsequent bacterial growth, especially in the early growth phase. The growth curves resemble those obtained for the $h m u Y$-deficient strain. The lack of inhibition of bacterial growth in the late growth phase may be caused by the expression of other iron/heme uptake systems important for $P$. gingivalis at this growth stage. In contrast, anti-HmuY antibodies demonstrated a greater ability to reduce biofilm formation since $P$. gingivalis cells pre-incubated with IgGs isolated from immune antiHmuY serum exhibited a lower ability of biofilm accumulation than the cells without added IgGs or pre-immune IgGs (figure 8). These data confirm that HmuY protein may be among the proteins important for biofilm accumulation by $P$. gingivalis.

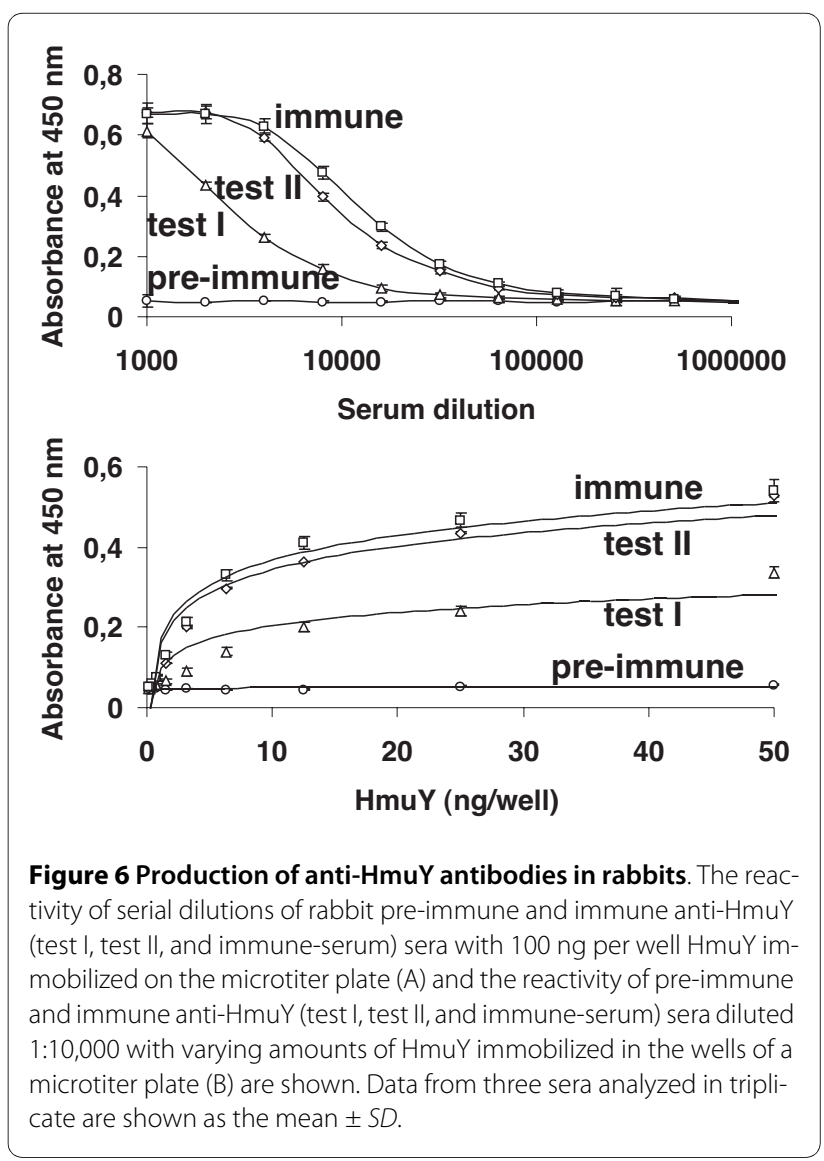




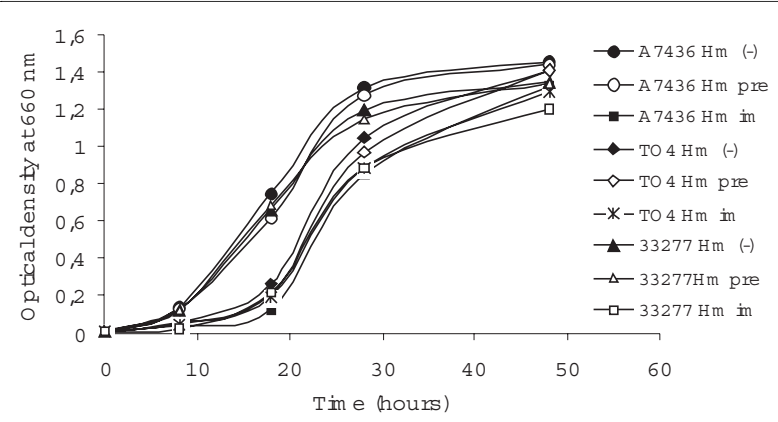

Figure 7 Inhibition of $P$. gingivalis growth by anti-HmuY IgG antibodies. The P. gingivalis wild-type A7436 and ATCC 33277 strains and the hmuY deletion mutant (TO4) strain were grown in basal medium supplemented with dipyridyl. The cells were then washed with PBS, incubated without lgGs (-), with purified pre-immune (pre), or immune (im) anti-HmuY IgGs and inoculated into fresh BM supplemented with hemin $(\mathrm{Hm})$.

\section{Conclusions}

As the prevalence of antibiotic-resistant strains of bacteria increases, novel ways of treating infections need to be developed. This is particularly important with respect to periodontal diseases, which are the most common chronic bacterial infections of man. First of all, HmuY may be important for a better understanding of the pathology caused by $P$. gingivalis. The surface exposure, high abundance, and immunogenicity of $P$. gingivalis HmuY protein suggest that its detailed examination may yield novel diagnostic methods. Knowledge of the molecular bases of the host immune response against $P$. gingivalis HmuY may be further essential for developing approaches to control and treat chronic periodontitis. To confirm these hypotheses, studies of anti-HmuY antibodies produced in patients with various forms of periodontal diseases and the influence of HmuY and anti-HmuY antibodies on the experimental periodontitis in a mouse model are now underway.

\section{Methods}

\section{Amino-acid sequence analyses}

HmuY homologues were identified using the Basic Local Alignment Search Tool (BLAST; http:// blast.ncbi.nlm.nih.gov/Blast.cgi) [44]. Prediction of signal peptides was performed with the LipoP 1.0 Server http:// www.cbs.dtu.dk/services/LipoP/[45]. Mature protein sequences were aligned using the CLUSTALW2 program [46] with the default alignment parameters: GONNET 250 protein weight matrix, gap opening penalty 10.00 , gap extension penalty 0.2 , penalty for closing a gap- 1 , and penalty for gap separation 4 . The phylogenetic tree was constructed with the neighbor-joining method [47]. Bootstrap analysis was performed using 1000 replicates with the CLUSTALW2 program. The tree was drawn with the NJplot program [48].

\section{Strains and growth conditions}

The $P$. gingivalis wild-type strains (A7436, W83, and ATCC 33277), the $h m u Y$ deletion mutant constructed in the A7436 strain (TO4), and the Bacteroides fragilis strain were grown anaerobically on blood agar plates (ABA; Biocorp), in Schaedler broth (Biocorp) and then cultured in basal medium alone (BM), BM supplemented with 1 $\mathrm{mg} / \mathrm{ml}$ hemin $(\mathrm{BM}+\mathrm{Hm}), 5 \%$ human serum $(\mathrm{BM}+$ serum $)$, or $160 \mu \mathrm{M}$ dipyridyl (BM+DIP) as described previously [19]. To avoid autolysis, the bacteria were grown for a time not exceeding $48 \mathrm{~h}$ [49]. E. coli cells were cultured as indicated in previous reports $[18,19]$.

\section{HmuY expression and purification}

P. gingivalis apo-HmuY lacking the first 25 residues (NCBI accession no. CAM 31898) was expressed using pHmuY11 plasmid and E. coli ER2566 cells (New England Biolabs) and purified from a soluble fraction of $E$. coli lysate as previously described [19]. The protein concentration was determined as previously reported [20].

\section{Immunization of rabbits}

A non-lipidated form of $\mathrm{HmuY}$ (the protein lacking the first 25 amino-acid residues comprising the signal peptide sequence, the following cysteine, and four additional amino acids, GKKK) was used to immunize rabbits (Lampire) with Freund's complete adjuvant. Purified HmuY (0.2 mg per injection) was injected subcutaneously. The animals were boosted on days $7,14,28,56$, and 84 of the immunization schedule and bled on days 1 (preimmune serum), 42 (test I serum), 70 (test II serum), and 98 (final-bleed immune serum). The IgG fraction was purified from serum using a HiTrap protein A column according to the manufacturer's instructions (Amersham Pharmacia).

\section{Protease accessibility assay}

To detect HmuY on the surface of the cell, wild-type (A7436, W83), hmuY-mutant (TO4), and E. coli cells over-expressing membrane-associated HmuY [19] were washed with $20 \mathrm{mM}$ sodium phosphate buffer, $\mathrm{pH}$ 7.6, containing $140 \mathrm{mM} \mathrm{NaCl}$ (PBS) and re-suspended in 50 $\mathrm{mM}$ Tris/ $\mathrm{HCl}, \mathrm{pH} 7.6$, containing $140 \mathrm{mM} \mathrm{NaCl}$ and 10 $\mathrm{mM} \mathrm{MgCl}$ to an optical density (OD) of 0.1 . The cell suspension was incubated with proteinase $\mathrm{K}(0.25 \mathrm{mg} / \mathrm{ml})$ for $30 \mathrm{~min}$ at $37^{\circ} \mathrm{C}$. After incubation, protease inhibitor cocktail (Complete; Roche) was added to stop the reaction, the cells were pelleted, suspended in PBS, and finally the samples were boiled in SDS-PAGE sample buffer. Then the proteins were separated by $15 \%$ SDS-PAGE and detected by Western blotting as described below. 

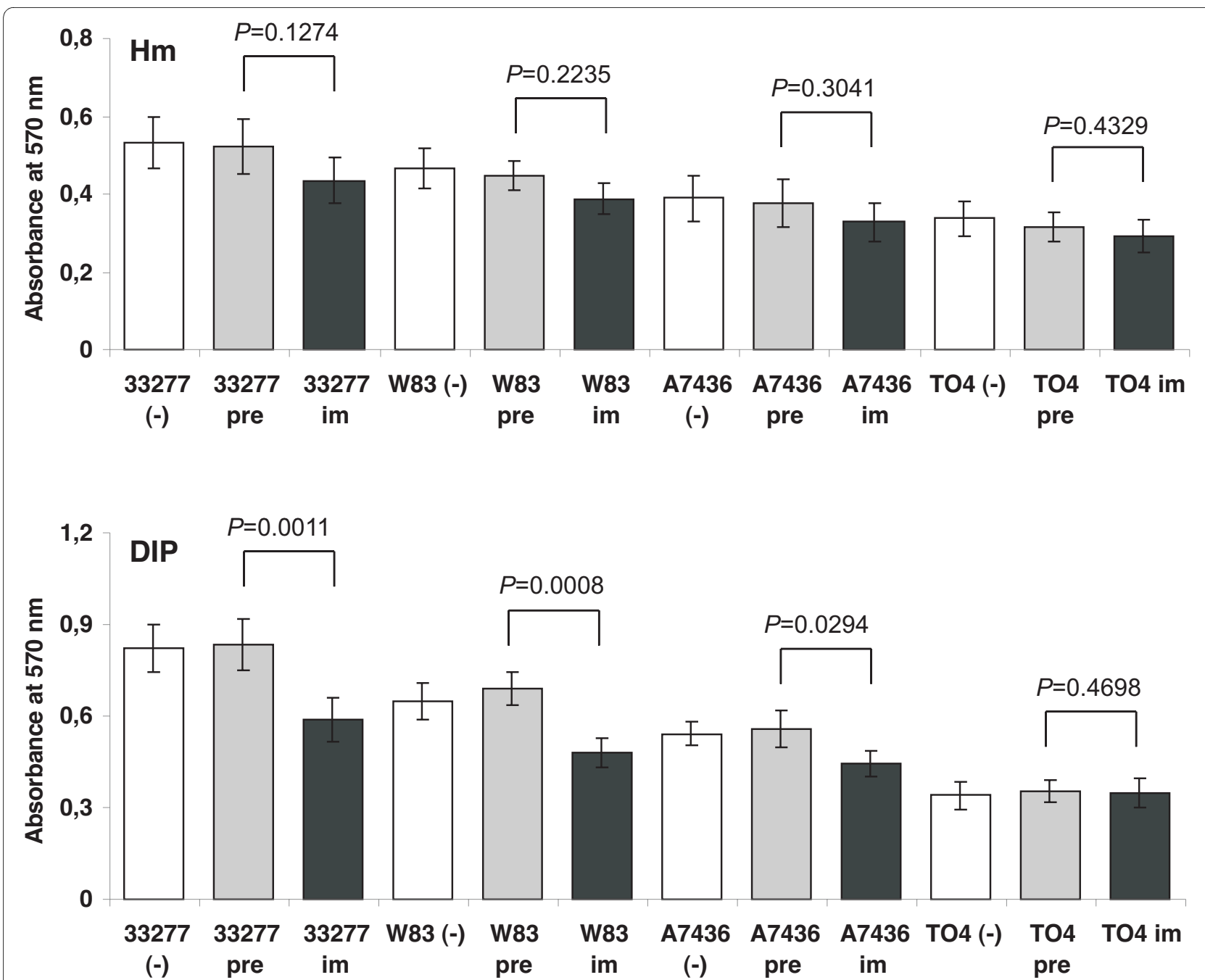

Figure 8 Inhibition of $P$. gingivalis biofilm formation by anti-HmuY IgG antibodies. P. gingivalis wild-type (A7436, W83, and ATCC 33277) strains and the hmuY deletion mutant strain constructed in A7436 (TO4) were grown in basal medium supplemented with hemin (Hm) or dipyridyl (DIP). The cells were washed with PBS, incubated with purified pre-immune or immune anti-HmuY IgGs, and inoculated into fresh media. The microtiter plate biofilms were stained with crystal violet. Data are shown as the mean $\pm S D$ of three independent experiments $(n=6)$. Differences between the cells incubated with pre-immune lgGs and cells incubating with immune anti-HmuY IgGs expressed as $p$ values are given above the respective bars.

Preparation of cells and proteins for SDS-PAGE and Western blotting

$P$. gingivalis cultures were centrifuged for $30 \mathrm{~min}$ at $20,000 \times g$ at $4^{\circ} \mathrm{C}$ and the supernatants were filtered through a $0.22-\mu \mathrm{m}$ pore-size filter (Roth). Bacterial pellets were washed with PBS and suspended in PBS to $\mathrm{OD}_{660}=0.1$. To separate outer-membrane vesicles, the filtered culture medium was centrifuged for $2 \mathrm{~h}$ at 100,000 $\times g$. For HmuY expression analysis, samples corresponding to $5 \mu \mathrm{l}$ of the bacterial culture at $\mathrm{OD}_{660}=0.1$ or $20 \mu \mathrm{l}$ of the culture medium were separated by $15 \%$ SDS-PAGE and transferred onto nitrocellulose membranes (Schleicher \& Schuell). Nonspecific binding sites were blocked with 5\% skim milk in PBS. HmuY was visualized with polyclonal anti-HmuY rabbit serum (Lampire) and secondary goat anti-rabbit IgG antibodies conjugated with horseradish peroxidase (HRP; Sigma), both used at 1:10,000 dilutions. The reaction was developed using chemiluminescence reagents (Western Lightning PlusECL; Perkin Elmer). To determine P. gingivalis autolysis, the presence of Fur was examined in both cells and culture medium using Western blotting with rabbit polyclonal antibodies raised against the synthetic peptide derived from the amino-acid sequence of Fur (CILADKDLRPPRFSY; GeneScript).

\section{Enzyme-Linked Immunosorbent Assay (ELISA)}

Levels of anti-HmuY antibodies in rabbit sera were determined by ELISA. For this purpose, 96-well polystyrene 
plates (Polysorp; Nunc) were coated for $1 \mathrm{~h}$ at $37^{\circ} \mathrm{C}$ with $100 \mu \mathrm{l} /$ well $\mathrm{HmuY}$ in PBS. The plates were washed three times with $200 \mu \mathrm{l}$ of PBS prior to blocking for $1 \mathrm{~h}$ at $37^{\circ} \mathrm{C}$ with $200 \mu \mathrm{l}$ of $2 \%$ bovine serum albumin (BSA) dissolved in PBS and then washed three times with $200 \mu \mathrm{l}$ of PBS. Two-fold serum dilutions or 1:10,000 serum dilutions (100 $\mu \mathrm{l}$ of pre-immune, test I, test II, and immune serum) were prepared in PBS and incubated for $1 \mathrm{~h}$ at $37^{\circ} \mathrm{C}$. After washing, antibody binding was detected using goat antirabbit IgG conjugated with HRP. After three final washes, a substrate solution $(100 \mu \mathrm{l})$ containing $0.05 \% o$-phenylenediamine (Sigma) with $0.01 \% \mathrm{H}_{2} \mathrm{O}_{2}$ was added for color development at room temperature. The reaction was stopped after $15 \mathrm{~min}$ by adding $25 \mu \mathrm{l}$ of $12.5 \% \mathrm{H}_{2} \mathrm{SO}_{4}$ and the absorbance was measured at $450 \mathrm{~nm}$ using a Multiskan Ascent microplate reader (Thermo Electron Corporation).

\section{Whole-cell ELISA, dot-blotting, and FACS analyses}

As an additional method of HmuY detection, cell surface staining with anti-HmuY antibodies was performed using whole-cell ELISA, dot-blotting, and flow cytometry (FACS) analyses. P. gingivalis cells grown to $\mathrm{OD}_{660}=1.0$ were used for these experiments. For the ELISA and dotblotting analyses, washed cells at several dilutions were adsorbed on the surface of microtiter plates or nitrocellulose membranes. Nonspecific binding of antibodies was prevented by incubation with $1 \%$ bovine serum albumin and $2 \%$ bovine fetal serum (Sigma) before the addition of rabbit pre-immune or anti-HmuY immune serum $(1: 10,000)$ or purified IgG fractions $(100 \mathrm{ng} / \mathrm{ml})$. After 1 -h incubation and washing with PBS, goat HRP-conjugated (Sigma) or bovine phycoerythrin-conjugated anti-rabbit IgG (Santa Cruz Biotechnology) at 1:10,000 or 1:500 dilutions were used, respectively. Finally, the cells, wells, and membranes were washed with PBS. For FACS analysis, the cells were fixed with $2 \% p$-formaldehyde. Then absorbance at $450 \mathrm{~nm}$ (ELISA), chemiluminescence (dot-blotting analysis), or fluorescence (FACS; Excalibur, Beckton Dickinson) were detected.

\section{Biofilm formation}

Homotypic biofilm formation by $P$. gingivalis was performed as described by others [50]. Briefly, $P$. gingivalis cells were grown on ABA plates, then in BM supplemented with hemin or dipyridyl to $\mathrm{OD}_{660}=1.0$ and used to inoculate fresh cultures to $\mathrm{OD}_{660}=0.1$. Cells in the appropriate medium were transferred $(200 \mu \mathrm{l})$ into sterile round-bottom microtiter plates (Sarstedt) and incubated under anaerobic conditions at $37^{\circ} \mathrm{C}$ for 24 or $48 \mathrm{~h}$. The resulting biofilms were washed with PBS, stained with $1 \%$ crystal violet, washed with PBS, and de-stained with $96 \%$ ethanol. Absorbance (A) was determined at $570 \mathrm{~nm}$ using a Multiskan Ascent microplate reader. The assays were repeated at least three times with each strain grown in eight wells. To confirm that the $P$. gingivalis cells were viable, the biofilm cells were scrapped into the respective medium and the OD at $660 \mathrm{~nm}$ and colony-forming unit (CFU) values were evaluated after 24 and $48 \mathrm{~h}$ (see Additional file 3). In parallel, bacteria were grown in planktonic form and the OD at $660 \mathrm{~nm}$ and CFU values were measured after 24 and $48 \mathrm{~h}$.

\section{Growth and biofilm inhibition studies}

Bacteria were grown overnight on ABA plates and then in $\mathrm{BM}$ supplemented with hemin or dipyridyl to $\mathrm{OD}_{660}=$ 1.0. After centrifugation, the bacteria were washed and suspended in PBS to $\mathrm{OD}_{660}=0.1$. Then $5 \mathrm{ml}$ of the bacterial suspension was centrifuged and the bacteria were incubated in $200 \mu \mathrm{l}$ of PBS for $1 \mathrm{~h}$ at $37^{\circ} \mathrm{C}$ with the IgG fraction purified from pre-immune or immune antiHmuY rabbit serum (200 ng). After addition of $5 \mathrm{ml}$ of the appropriate medium, planktonic bacterial growth was monitored by measuring the OD at $660 \mathrm{~nm}$ or biofilm formed as described above. Assays were performed three times in duplicate.

\section{Statistical analysis}

Data are expressed as means values \pm standard deviations (mean $\pm S D$ ). Statistical analysis was performed using unpaired Student's $t$ test (GraphPad Prism 5). Values of $p$ $<0.05$ were considered statistically significant.

\section{Additional material}

\footnotetext{
Additional file 1 Comparison of HmuY homologues. Comparison of

homologous HmuY amino-acid sequences identified in human pathogens (A) and bacteria identified in oral tissues (B). Amino-acid sequences lacking signal peptides are shown. Positions with identical amino acids in more than $30 \%$ of the sequences are shown in black boxes and partial homology is indicated in grey boxes. Phylogenetic relationship between homologous HmuY amino-acid sequences (C). Bacteria infecting the oral cavity are shown in bold. The phylogenetic tree was determined with the NeighborJoining method. Bootstrap values are included. Pgi, Porphyromonas gingivalis; Pen, P. endodontalis; Pue, P. uenonis; Bfr, Bacteroides fragilis; Bfi, B. finegoldii; $\mathrm{Bco}$, B. coprocola; Bst, B. stercoris; Bdo, B. dorei; Bvu, B. vulgatus; Bov, B. ovatus; Bca, B. caccae; Bth, B. thetaiotaomicron; Bcp, B. coprophilus; Bsp, Bacteroides sp.; Coc, Capnocytophaga ochracea; Cgi, C. gingivalis; Csp, C. sputigena; Lbo, Leptospira borgpetersenii; Lin, L. interrogans; Ssp, Sphingobacterium spiritivorum; Pbi, Prevotella bivia; Por, P. oris; Pbe, P. bergensis; Pti, P. timonensis; Pme, P. melaninogenica; Pve, P. veroralis; Psp, Prevotella sp.; Pta, P. tannerae.

Additional file $\mathbf{2}$ Analysis of surface exposure of HmuY. Analysis of surface exposure of $P$. gingivalis HmuY analyzed by whole-cell ELISA. P. gingivalis wild-type (A7436, W83) and hmuY deletion mutant (TO4) strains were grown in basal medium supplemented with hemin ( $\mathrm{Hm}$ ) or dipyridyl (DIP). The cells were washed and diluted with PBS (starting at $\mathrm{OD}_{660}=1.0$ ). Varying dilutions of $P$. gingivalis cells were adsorbed on the wells of the microtiter plate and reacted with pre-immune serum (A) or purified pre-immune IgGs (pre) (B) and immune anti-HmuY serum (A) or purified immune antiHmuY IgGs (im) (B). Representative data are shown.

Additional file $3 P$. gingivalis growth in broth cultures and biofilms, and biofilm accumulation. P. gingivalis growth was analyzed by measuring the $\mathrm{OD}$ at $660 \mathrm{~nm}$, cell viability by plating cells on ABA plates and colony forming unit (CFU) calculation, and biofilm accumulation by microtiter plate assay
} 


\section{Authors' contributions}

TO conceived the study, contributed to its design, laboratory experiments, and data analysis and wrote the manuscript. HW, JC, and MO contributed to the design, laboratory experiments, and the writing of the manuscript. All authors have read and approved the final manuscript.

\section{Acknowledgements}

This work was supported in part by grant nos. N401 029 32/0742, N N303 406136, and N N303 518438 from the Ministry of Science and Higher Education, and by Wroclaw Research Center EIT+ under the project "Biotechnologies and advanced medical technologies - BioMed" (POIG 01.01.02-02-003/08/00) financed from the European Regional Development Fund (Operational Program Innovative Economy, 1.1.2) (TO) and the European Social Fund (Human Capital Program, 8.2.2), state, and province government under the project "Grant - support of research through scientific stipends for PhD students" (HW).

\section{Author Details}

Laboratory of Biochemistry, Faculty of Biotechnology, University of Wroclaw, Tamka 2, 50-137 Wroclaw, Poland

Received: 6 October 2009 Accepted: 4 May 2010

Published: 4 May 2010

\section{References}

1. Pihlstrom BL, Michalowicz BS, Johnson NW: Periodontal diseases. Lancet 2005, 366:1809-1820.

2. Schenkein HA: Host responses in maintaining periodontal health and determining periodontal disease. Periodontol 2000, 200640:77-93.

3. Mayrand D, Holt SC: Biology of asaccharolytic black-pigmented Bacteroides species. Microbiol Rev 1988, 52:134-152.

4. Lamont RJ, Chan A, Belton CM, Izutsu KT, Vasel D, Weinberg A: Porphyromonas gingivalis invasion of gingival epithelial cells. Infect Immun 1995, 63:3878-3885.

5. Belton CM, Izutsu KT, Goodwin PC, Park Y, Lamont RJ: Fluorescence image analysis of the association between Porphyromonas gingivalis and gingival epithelial cells. Cell Microbiol 1999, 1:215-223.

6. Progulske-Fox A, Kozarov E, Dorn B, Dunn W Jr, Burks J, Wu Y: Porphyromonas gingivalis virulence factors and invasion of cells of the cardiovascular system. J Periodontol Res 1999, 34:393-399.

7. Bartold PM, Marshall RI, Haynes DR: Periodontitis and rheumatoid arthritis: a review. J Periodontol 2005, 76:2066-2074.

8. Leon R, Silva N, Ovalle A, Chaparro A, Ahumada A, Gajardo M, Martinez M, Gamonal J: Detection of Porphyromonas gingivalis in the amniotic fluid in pregnant women with a diagnosis of threatened premature labor. $J$ Periodontol 2007, 78:1249-1255.

9. Mattila KJ, Pussinen PJ, Paju S: Dental infections and cardiovascular disease: a review. J Periodontol 2005, 76:2095-2088.

10. Wang Q, Zhou X, Huang D: Role for Porphyromonas gingivalis in the progression of atherosclerosis. Med Hypotheses 2009, 72:71-73.

11. McKee AS, McDermid AS, Baskerville A, Dowsett AB, Ellwood DC, Marsh PD: Effect of hemin on the physiology and virulence of Bacteroides gingivalis. Infect Immun 1986, 52:349-355.

12. Olczak T, Simpson W, Liu X, Genco CA: Iron and heme utilization in Porphyromonas gingivalis. FEMS Microbiol Rev 2005, 29:119-144.

13. Liu X, Olczak T, Guo HC, Dixon DW, Genco CA: Identification of essential amino acid residues required for hemoprotein utilization in the Porphyromonas gingivalis heme receptor HmuR. Infect Immun 2006, 74:1222-1232

14. Olczak T: Analysis of conserved glutamate residues in Porphyromonas gingivalis heme receptor HmuR: toward a further understanding of heme uptake. Arch Microbiol 2006, 186:393-402.

15. Olczak T, Dixon DW, Genco CA: Binding specificity of the Porphyromonas gingivalis heme and hemoglobin receptor $\mathrm{HmuR}$, gingipain $\mathrm{K}$, and gingipain R1 for heme, porphyrins, and metalloporphyrins. J Bacterio/ 2001, 183:5599-5608.

16. Simpson W, Olczak T, Genco CA: Characterization and expression of HmuR, a TonB-dependent hemoglobin receptor of Porphyromonas gingivalis. J Bacteriol 2000, 182:5737-5748.

17. Lewis JP, Plata K, Fan Y, Rosato A, Anaya C: Transcriptional organization, regulation and role of the Porphyromonas gingivalis W83 hmu haeminuptake locus. Microbiology 2006, 152:3367-3382.
18. Olczak T, Siudeja K, Olczak M: Purification and initial characterization of a novel HmuY protein from Porphyromonas gingivalis expressed in Eschericha coli and insect cells. Protein Expr Purif 2006, 49:299-306.

19. Olczak T, Sroka A, Potempa J, Olczak M: Porphyromonas gingivalis HmuY and HmuR - further characterization of a novel mechanism of heme utilization. Arch Microbiol 2008, 183:197-210

20. Wojtowicz H, Wojaczynski J, Olczak M, Kroliczewski J, Latos-Grazynski L, Olczak T: Heme environment in HmuY, the heme-binding protein of Porphyromonas gingivalis. Biochem Biophys Res Commun 2009, 383:178-182.

21. Wojtowicz H, Guevara T, Tallant C, Olczak M, Sroka A, Potempa J, Sola M, Olczak T, Gomis-Ruth FX: Unique structure and stability of HmuY, a novel heme-binding protein of Porphyromonas gingivalis. PLOS Pathogens 2009, 5:e100041.

22. Wolff N, Izadi-Pruneyre N, Couprie J, Habeck M, Linge J, Rieping W, Wandersman C, Nilges M, Delepierre M, Lecroisey A: Comparative analysis of structural and dynamic properties of the loaded and unloaded hemophore HasA: functional implications. J Mol Bio/ 2008, 376:517-525.

23. Garrity GM, Bell JA, TG Lilburn: Taxonomic outline of the prokaryotes release 5.0 May 2004. In Bergey's manual of systemic bacteriology Springer-Verlag, New York; 2004

24. Kumar PS, Griffen AL, Moeschberger ML, Leys EJ: Identification of candidate periodontal pathogens and beneficial species by quantitative $16 \mathrm{~S}$ clonal analysis. J Clin Microbiol 2005, 43:3944-3955.

25. Riep B, Edesi-Neuss L, Claessen F, Skarabis H, Ehmke B, Flemming TF, Bernimoulin JP, Gobel UB, Moter A: Are putative periodontal pathogens reliable diagnostic markers? J Clin Microbiol 2009, 47:1705-1711.

26. Sigueira JF Jr, Rocas IN, Alves FR, Silva MG: Bacteria in the apical root canal of teeth with primary apical periodontitis. Oral Surg Oral Med Oral Pathol Oral Radiol Endod 2009, 107:721-726.

27. Brito LCN, Teles FR, Franca EC, Ribeiro-Sobrinho AP, Haffajee AD, Socransky SS: Use of multiple-displacement amplification and checkerboard DNA-DNA hybridization to examine the microbiota of endodontic infections. J Clin Microbiol 2007, 45:3039-3049.

28. Masakiyo Y, Yoshida A, Shintani Y, Takahashi Y, Ansai T, Takehara T: The identification of genes specific to Prevotella intermedia and Prevotella nigrescens using genomic subtractive hybridization. Anaerobe 2009. doi: 10.1016/j.anaerobe.2009.11.003

29. Colombo AP, Boches SK, Cotton SL, Goodson JM, Kent R, Haffajee AD, Socransky SS, Hasturk H, Van Dyke TE, Dwehirst F, Paster BJ: Comparisons of subgingival microbial profiles of refractory periodontitis, severe periodontitis, and periodontal health using the human oral microbe identification microarray. J Periodontol 2009, 80:1421-1432.

30. Haraldsson G, Holbrook WP: Identifying clinically important gramnegative anaerobes from the oral cavity. Eur J Oral Sci 1999, 107:429-436.

31. Riggio MP, Aga CA, Murray CA, Jackson MS, Lennon A, Hammersley N, Bagg J: Identification of bacteria associated with spreading odontogenic infections by $16 \mathrm{~S}$ rRNA gene sequencing. Oral Surg Oral Med Oral Pathol Oral Radiol Endod 2007, 103:610-617.

32. Babu MM, Priya ML, Selvan AT, Madera M, Gough J, Aravind L, Sankaran K: A database of bacterial lipoproteins (DOLOP) with functional assignments to predicted lipoproteins. J Bacteriol 2006, 188:2761-2773.

33. Mihara J, Holt SC: Purification and characterization of fibroblastactivating factor isolated from Porphyromonas gingivalis W50. Infect Immun 1993, 61:588-595.

34. Mazmanian SK, Skaar EP, Gaspar AH, Humayun M, Gornicki P, Jelenska J, Joachmiak A, Missiakas DM, Schneewind O: Passage of heme-iron across the envelope of Staphylococcus aureus. Science 2003, 299:906-909.

35. Ang CS, Veith PD, Dashper SG, Reynolds EC: Application of ${ }^{16} \mathrm{O} /{ }^{18} \mathrm{O}$ reverse proteolytic labeling to determine the effect of biofilm culture on the cell envelope proteome of Porphyromonas gingivalis W50. Porphyromonas gingivalis 2008, 8:1645-1660.

36. Dashper SG, Ang CS, Veith PD, Mitchell HL, Lo AW, Seers CA, Walsh KA, Slakeski N, Chen D, Lissel JP, Butler CA, O'Brien-Simpson NM, Barr IG, Reynolds EC: Response of Porphyromonas gingivalis to heme limitation in continuous culture. J Bacterio/ 2009, 191:1044-1055

37. Lo AW, Seers CA, Boyce JD, Dashper SG, Slakeski N, Lissel JP, Reynolds EC: Comparative transcriptomic analysis of Porphyromonas gingivalis biofilm and planktonic cells. BMC Microbiology 2009, 9:18. 
38. Wu J, Lin $X, X i e H$ : Regulation of hemin binding proteins by a novel transcriptional activator in Porphyromonas gingivalis. J Bacteriol 2009, 191:115-122.

39. Costerton JW, Stewart PS, Greenberg EP: Bacterial biofilms: a common cause of persistent infections. Science 1999, 284:1318-1322.

40. Socransky SS, Haffajee AD, Cugini MMA, Smith C, Kent RL Jr: Microbial complexes in subgingival plaque. J Clin Periodontol 1998, 25:134-144

41. Chung WO, Park Y, Lamont RJ, McNab R, Barbieri B, Demuth DR: Signaling system in Porphyromonas gingivalis based on a LuxS protein. J Bacteriol 2001, 183:3903-3909.

42. James $C E$, Hasegawa Y, Park Y, Yeung V, Tribble GD, Kuboniwa M, Demuth $D R$, Lamont RJ: LuxS involvement in the regulation of genes coding for hemin and iron acquisition systems in Porphyromonas gingivalis. Infect Immun 2006, 74:3834-3844

43. McNab R, Ford SK, El-Sabaeny A, Barbieri B, Cook GS, Lamont RJ: LuxSbased signaling in Streptococcus gordonii: autoinducer 2 controls carbohydrate metabolism and biofilm formation with Porphyromonas gingivalis. J Bacteriol 2003, 185:274-284.

44. Altschul SF, Madden TL, Schaffer AA, Zhang J, Zhang Z, Miller W, Lipman DJ: Gapped BLAST and PSI-BLAST: a new generation of protein database search programs. Nucleic Acids Res 1997, 25:3389-3402.

45. Juncker $S$, Willenbrock $H$, von Heijne $G$, Nielsen $H$, Brunak S, Krogh A: Prediction of lipoprotein signal peptides in Gram-negative bacteria. Protein Sci 2003, 12:1652-1662.

46. Larkin MA, Blackshields G, Brown NP, Chenna R, McGettigan PA, McWilliam $H$, Valentin F, Wallace IM, Wilm A, Lopez R, Thompson JD, Gibson TJ, Higgins DG: ClustalW and ClustalX version 2. Bioinformatics 2007, 23:2947-2948

47. Saitou N, Nei M: The neighbor-joining method: a new method for reconstructing phylogenetic trees. Mol Biol Evol 1987, 4:406-425.

48. Perriere G, Gouy M: WWW-Query: An on-line retrieval system for biological sequence banks. Biochimie 1996, 78:364-369.

49. Kamaguchi A, Nakano M, Shoji M, Nakamura R, Sagane Y, Okamoto M, Watanabe T, Ohyama T, Ohta M, Nakayama K: Autolysis of Porphyromonas gingivalis is accompanied by an increase in several periodontal pathogenic factors in the supernatant. Microbiol Immunol 2004, 48:541-545

50. Capestany CA, Kuboniwa M, Jung IY, Park Y, Tribble GD, Lamont RJ: Role of the Porphyromonas gingivalis InIJ protein in homotypic and heterotypic biofilm development. Infect Immun 2006, 74:3002-3005.

doi: 10.1186/1471-2180-10-134

Cite this article as: Olczak et al., Species specificity, surface exposure, protein expression, immunogenicity, and participation in biofilm formation of Porphyromonas gingivalis HmuY BMC Microbiology 2010, 10:134

Submit your next manuscript to BioMed Centra and take full advantage of:

- Convenient online submission

- Thorough peer review

- No space constraints or color figure charges

- Immediate publication on acceptance

- Inclusion in PubMed, CAS, Scopus and Google Scholar

- Research which is freely available for redistribution

Submit your manuscript at www.biomedcentral.com/submit
C Biomed Central 